\title{
The Potential for Virtual Communities To Promote Diversity in the Sciences
}

Published version of this pre-print is: Murthy, Dhiraj, Rodriguez, Atilano and Kinstler, Linda. 2013. The potential for virtual communities to promote diversity in the sciences. Current Sociology, 61(7), pp. 1003-1020. ISSN 0011-3921 http://dx.doi.org/10.1177/0011392113502994

\section{Introduction}

Women have been historically underrepresented in scientific fields around the world. Despite the concerted efforts of UNESCO, the EU, and other prominent international institutions over the past several decades (Marin 2007: 15) to improve the representation of women in the highest levels of academia and research labs around the world, "there is a clear pattern of female underrepresentation everywhere" (European Commission 2012). In the US, for example, women account for $50 \%$ of M.D. degrees and $52 \%$ of doctorates in the life sciences-and earn over $50 \%$ of doctorate degrees across all disciplines - but only represent $25 \%$ of tenured faculty positions in the United States (Ceci \& Williams 2011; Gee \& Norton 2009). In Europe, the number of women earning doctorate degrees has also increased to over 50\% in 12 of 26 EU Member States, but women occupied only 33\% of research positions as of 2009 (EU Commission 2012: 2). The imbalance is currently so drastic that in its 2012 "She Figures" report, the European Commission concluded, "despite a number of positive trends, women in scientific research remain a minority, segregation across scientific fields is strong and the glass ceiling continues to hold back women from top academic positions" (EU Commission 2012: 2). In 2007, the European Union began developing a transnational online network, eument-net, which would connect five academic institutions in a virtual consortium specifically geared toward providing mentors for women in the sciences (Nöbauer \& Ganetti 2008: 10). The issue received renewed attention following the Lisbon summit of 2000, when EU leaders agreed upon a strategy to achieve "a substantial increase in women's participation at all levels of higher education and research" (Nöbauer \& Ganetti 2008: 66-7). Increasing mentoring resources for women in the sciences is also a primary objective of the ENWISE (Enlarge Women in Science to East) Expert Group, which was created to promote gender equality in second-wave EU countries in the Baltics and eastern and central Europe (EU Commission 2003).

The continued underrepresentation of women in the sciences has been attributed to 
discrimination on the basis of gender, but recent studies have found that women are underrepresented in the sciences not simply because of biased grant agencies, journals, and hiring committees, but rather as a result of "factors surrounding family formation and childrearing, gendered expectations, lifestyle choices, and career preferences-some originating before or during adolescence" (Ceci \& Williams 2011: 3161). Balancing personal and professional life is a primary obstacle for women in the sciences, though gender bias persists in academia (Moss-Racusin 2012: 16476) and impacts the development of mentoring relationships between young female scientists and their professors. A comparative study of men and women chemists confirmed the thesis of Social Cognitive Career Theory, which argues that individual perceptions of career opportunities affect professional decision making: women reported lower levels of mentoring than their male colleagues at all levels of education (Nolan 2008: 238). In a study of OURNET, a listserv dealing with issues of women in the sciences, Kleinman (2008) found that members were inhibited by "social norms or barriers [that] prevent them from seeking information, advice, or support from their workplace colleagues" (Kleinman 2003: 87). The emergence of virtual forums specifically aimed at addressing real-world obstacles that women in the sciences face suggests that online communities provide unique discursive spaces for empowering underrepresented groups. The emergence of these positive virtual spaces is particularly important to note given that many spaces and interactions on the Internet reaffirm, reinscribe, or magnify gender inequalities. For example, a study of online scholarly exchanges between academics in Southeast Asia and the US found that Internet-mediated interactions do not "necessarily "level the playing field" [... and may] even propagate or exacerbate the inherent positional inequalities in the system' (Thompson 2006: 55). For example, blogshops, fashion blogs that sell women's clothing and accessories, were touted to be empowering the women who ran them, but have been found to "play out an internalized "male gaze" and "conform to sociocultural expectations of emphasized femininity' (Abidin and Thompson: 474).

This paper focuses on WomenScientists1, an online forum within a larger life science community of practice. The forum has a global reach, but most participants are affluent, white women based primarily in the UK and US. 171 unique users have participated in WomenScientists1. Users are asked to identify themselves, at a minimum, only with a username of their choice. The forum is geared toward issues relevant to female scientists. Through an empirical study of forum posts, profiles, and other forum data, this paper finds that there is a real 
problem of women participating in the sciences and that online mentoring offers discernible solutions to address this problem. Ultimately, WomenScientists1, was found to be a positive and supportive space for women scientists to virtually interact and mentor each other. It was found to create support, solidarity, and identity amongst its members.

\section{Virtual Communities and Social Inequalities}

Sassen (2002: 370) argues, Internet "use is constructed or constituted in terms of specific cultures and practices through and within which users articulate the experience/utility of electronic space". Ultimately, virtual communities are gendered, shaped by "mediating cultures" that interpellate online actors through social, political, and economic terms. Though Internet traffic is expected to quadruple by 2015 (Cisco 2011), access to the Internet in the developing world remains largely confined to urban elites. Where emergent online communities are forming, however, the literature shows they are providing new, inclusive spaces for underrepresented minorities to communicate and solidify group identity. Virtual communities have been researched broadly within the context of underrepresented groups. For example, UNESCO observes that Information and Communication Technologies (ICT) can facilitate greater representation of women in the public sphere because online communication provides "simple access to information and improved communications [which] can end the isolation of women" (Huyer and Sikoska 2003: 5). This stems from the view that the Internet can serve as an equalizer by disseminating information and invigorating discussions amongst different diverse individuals regardless of the biases they face. By mediating these spaces, the Internet has the potential to increase representation. (Papacharissi 2008).

Virtual communities are constitutive as well as empowering; writing in this journal, Brickell (2012) has examined how individuals are constituted as subjects on the Internet (through Althuserian and Foucauldian modes of analysis). His research interrogates the influence of symbolic interactionism in new media studies, and argues that dynamically networked individuals constitute their identities through the architecture and content of virtual communities. Conceived as such, online forums become virtual spaces designated for the creation of new discourses of gender and sexuality. They may enable underrepresented groups to enunciate and constitute collective identity in a way that may be difficult face-to-face. Studies on Internet use 
among low-income families, sexual minorities, and African-American women have found that online networks can only be used for social empowerment when they address the practical realities of everyday life and break the cycle of "regard[ing] the use of the internet as separate from the social realities of disadvantaged user communities" (Mehra \& Merkel 2004: 799). In a previous issue of this journal, Arora (2012) situates emerging virtual modes of interactivity within "real-world infrastructures," investigating how individual actors enact identity online. Digital inequalities, Schulz (2012: 248) argues, stem from pre-existing social inequities. Often, this leads to the creation of customized online spaces in which people interact with those who share a specific interest, culture, or identity. The structure of virtual communities determines the parameters by which Internet users identify themselves within specialized groups-social networks ask for a wide range of information and give users varying degrees of freedom in deciding how much information they would like to provide. As Brickell (2012: 31) puts it, users "may be simultaneously enabled and limited by the architectural factors of the website, the material circumstances of their lives and the impressions formed by cyberspace's other inhabitants".

\section{The Impact of Online Mentoring}

Women in science, technology, engineering, and math (known as STEM fields in the US and STS - Science, Technology, and Society_in Europe) are forming virtual communities to discuss the gendered aspects of scientific professions, and online forums are helping shape mentoring networks for young female scientists. However, this trend is by no means limited to the sciences. For instance, Stein (2009: 4) found that women in legal professions are turning to blogging "to resolve their disputes, address their personal grievances, challenge implicit male bias engrained in the profession, and share and obtain the information they need to become stronger bargainers in the workplace." Online networks and their concomitant public spheres are unique spaces in which women can discuss the impact of their professions on their personal lives in language that is often harder to enunciate face-to-face. Working environments often preclude intimate discussion of this kind, unintentionally encouraging women to turn to virtual communities for support. Cole (2011: 1161) examines how women with disabilities used the online forum GimpGirl Community to develop a community discourse that challenges existing 
norms of femininity. Kennedy (2000) observes how women interacted online and how their interactions created a 'sense of community' through virtual forums.

Online spaces have the potential to further discourse amongst women both professionally and personally. As Kennedy (2000) demonstrates, women tend to construct such virtual communities because they want to communicate with others who share their experiences or have overcome similar gender barriers. Zorn's (2004) study of Vifu (Virtual International Women's University) substantiates this conclusion: Vifu allowed women from diverse global backgrounds to come together to discuss relevant issues, and Zorn, like Kennedy (2000) argues that the strategic actions that arose from the discussions on the site fostered a strong sense of community amongst the users. Fay (2007) describes the goals that the coordinator of vifu had in that '[i]t was imagined as a 'lived in' space, a truly user friendly platform for networking in which the focus was on 'being together' rather than technological know-how' (Fay 2007: 5). Online spaces geared toward women promote the formation of a group identity amongst their users and serve as environments where participants can communicate with and support one another.

Recent studies (Nolan 2008, Moss-Racusin 2012) have found that the relationship between female scientists and their professors is a reliable indicator of future success: the higher the quality of mentorship received, the more likely it is that female scientists will advance in their respective fields. A healthy mentoring relationship can be instrumental in inspiring women to complete their degree (Chesler and Chesler, 2002). Preston (2004: 92) concludes that without these mentoring relationships, women are more likely to drop out of the STEM and STS pipelines, and that insufficient levels and quality of mentoring adversely affects female scientists more than the same deficiencies would hinder male scientists. Secondly, an understanding of the issues that women face in the sciences proves crucial to establishing and maintaining a positive mentoring relationship. "Women in academic settings [...] were less likely than men to be mentored, possibly because of the small number of potential mentors who understand the needs of a woman in science and possibly because of potential mentors' differing expectations about the success of men and women in science (Preston 2004: 110). Indeed, Moss-Racusin (2012: 16476) found that male and female professors were equally likely to demonstrate a subtle bias against female scientists. As Kleinman (2000) and Single et al. (2005) conclude, electronically mediated communication focusing on women's issues is especially influential for women who most stand to benefit from advice on overcoming gender-specific hardships. 
A number of virtual communities have already emerged as vital spaces for women to access mentoring networks. For instance, the University of Southern Queensland's GoWEST Project created mentoring relationships between undergraduates and scientists with higher degrees, combining offline and virtual communication (Cater-Steel and McDonald 2011). Single et al. (2000: 125) discuss the success of MentorNet, an online network that matches women in STEM and STS fields with prospective mentors. Members were aware that readers of their posts would "fulfill their need to interact in a safe and comfortable setting with other women professional engineers and scientists, and sympathetic men" (Single et. al. 2000:125). MentorNet's virtual space was found to augment traditional mentoring, as users who had a mentor used MentorNet in addition to email to communicate with one another (Single et al. 2005). The overall result was that the mentees experienced increases in self-confidence, which enabled them to feel more secure about their abilities in their respective scientific fields.

Kleinman (2003) describes how members of the listserv OURNET, which is geared toward answering questions pertaining to the role of women in science and engineering, found that their self-confidence was boosted by taking an active role in the forum. "When they helped other OURNET members through information exchange and emotional support communicated through public or private e-mail messages, they were doing something that they believed in, and it made them feel good about themselves" (Kleinman 2003: 80). Users who directly responded to specific posts felt more connected to the network and gained reflexive satisfaction from their contribution. Kleinman's study concludes that the forum's computer-mediated communication offers a variety of different experiences for the members of the community that address the individual needs of OURNET members.

\section{WomenScientists1}

WomenScientists 1 is an online forum within a larger life science community of practice. Created in February 2009, the forum serves as a virtual space for supporting women and promoting gender diversity in the sciences. Those who belong to the forum are designated either as users or members; users are those individuals who belong to the parent site of WomenScientists1, while members are those who subscribe specifically to the forum. In other words, members are users specifically subscribed to this forum. Members of the forum do not all 
regularly contribute to the group and the users who post are not necessarily members of the forum. WomenScientists 1 consists of 39 members with 171 unique users having posted to the forum. 71 non-member users posted only to this specific forum and not to any other discussion on the parent site. The majority of users with sex identified on their profile are women: 53 women and 17 men belong to the forum (not all are members and not all contributors reported their sex). Additionally, the majority of individuals who reported their location, identified as living in Great Britain, with the United States following second. From the information posted to the forum and from profiles, we found that members of WomenScientists 1 are employed in a range of fields, including academia, journalism, and information systems. Of 23 members who reported their race, 17 identified as white and six identified as non-white, and the majority of users who reported their age fell between 18 and 40 years old - a critical age group as "the formative predoctoral years are a critical window, because students' experiences at this juncture shape both their beliefs about their own abilities and subsequent persistence in science" (MossRacusin et al. 2012: 16475). Of those who provided their level of education, $81 \%$ had at least a masters degree. The majority of contributors to WomenScientists 1 are highly educated, relatively affluent women based primarily in the UK and US. The moderator of WomenScientists 1 is a user who works as a journalist but has a Masters degree in a science field. As of the time of writing, the forum consisted of 65 topics with a total of 325 replies.

Forums in this virtual community typically have a common theme, serve as social spaces for group formation, and host active, subject-specific discussions. Our research focused on the aggregate material of the WomenScientists1 forum, which provided a middle ground between the broad subject matter of the parent site and the narrow focus of topics within the forum. Because the forum does not require users to verify their identities or provide biographical information, it can serve as a safe place where individuals feel they can speak more freely. In this sense, WomenScientists1 shares a characteristic typical of blogs, which often demonstrate a 'tension between visibility and invisibility [that] gives blogging a confessional quality, where a less polished and even uglier self can be verbalized' (Hookway 2008: 96-97), and thus more accurately address issues facing women in scientific fields. Ross (2005: 343-4 cited in Brickell) has found that the freedom of forum members to maintain their anonymity can be a form of empowerment that "reside[s] in the individual's ability to change form, age, gender, position, or sexual orientation" online. Additionally, withholding personal information helps to ensure that 
the colleagues of forum contributors will not know of their online activity. This reduces the possibility that the identities of forum members will be recognized, and points to a perceived need to maintain anonymity to avoid being penalized for their contributions in the workplace. Because of this, women would also ask questions and mentor one another on issues such as motherhood in science, work/family life balance, and glass ceilings.

\section{Methods}

Online forums have been successfully studied using symbolic interactionist perspectives (Williams and Copes, 2005). Our study considers that virtual communities are an 'evolving process' that can be examined using symbolic interactionism (Fernback 2007). Additionally, we draw from Robinson's (2007) approach of using symbolic interactionism to help decipher a “cyber I, 'me", and a more nebulous "digital 'generalized' other". Like most web-based forums, WomenScientists 1 is asynchronous and the space is heavily textual. Given these characteristics, we followed one of Williams' and Copes' (2005: 74) methods of studying online forums, which argues for 'textual content analysis of forum threads without focused interaction with participants'.

Like Williams and Copes (2005), coding for forums was conducted using QSR NVivo and employed emergent coding (Hutchison et al., 2009). To ensure proper coding of the text for several different characteristics of the community, we used 22 unique codes with 12 tree nodes and 10 free nodes. The free nodes and tree nodes (including their children) are described in detail in Tables 1 and 2. Codes were explained to all researchers in our team in order to ensure accuracy and a common understanding of what each code signified. We developed a data scraping application specifically for the purpose of collecting forum data, and received the organization's written permission to conduct our research. The application was designed to record details of each post, including author name, date of post, and the structure of the discussion (i.e. preserving the threads and sub-threads). Each post was coded according to content: "Answers" were those posts that responded to a previous question, while posts coded "Asks" were those positing a question and soliciting a response. The codes took account of the general subject matter of each post: codes included "Personal," in which a member offers personal information or experience, "Career" (relating to professional advancement), "Stigma- 
Women" (addressing setbacks specific to women in science), "Support" (agreeing with or affirming a previous comment), "Science Speak" (posts using technical language), and others as noted in Table 1.

\section{INSERT TABLE 1 HERE}

Table 1: Free Nodes used for coding

Throughout the study, the posts recorded from WomenScientists1 have been altered to minimize the possibility of identification, while preserving their original intention. Following the example of Kraut et. al. (2004: 109), some text was altered to reduce the probability that forum contributors could be identified by a simple online search for personal statements included in the study. Although the posts can be accessed online, these precautions reduce identification possibilities. Kleinman (2003) cites an example of a user requesting to use a pseudonym and preferring to sending private messages in order to avoid developing an online reputation as a feminist, which she believed would cause her to be stigmatized by co-workers should they come across her online posts. In deference to these concerns, the altered quotations in this study maintain the spirit of the original message rather than the letter.

\section{INSERT TABLE 2 HERE}

Table 2: Parent Tree Nodes used for coding

Beyond coding for content alone, our application coded interactions between active users in order to trace the sentiments conveyed between individuals belonging to the same virtual community. One problem that arose with our coding algorithm was the fact that sometimes users would not name the author to which they were responding, choosing instead to refer to the idea of a previous post and therefore creating room for ambiguity. In order to code for this type of interaction, as per Kim and Hovy's (2006) recommendation, we designated the category of "general reference," which indicates a weaker connection between users who did not directly address one another. The main categories used to code responses were "positive," "neutral," and "negative," and the "general reference" qualifier added to these categories, and lastly the unrelated category. A "positive" post is one where a user shows strong support for a previous post while a "negative" post is one where the user shows opposition to a previous post or position. Neutral posts lacked a sense of support or opposition to a previous post. Lastly, posts 
coded as "unrelated" were those that did not pertain to the conversation taking place. Neviarouskaya et.al. (2007: 228) argues that in attempting to capture the nature of a continuous discussion, "we should also take into account the emotion dynamics throughout the conversation, or its, 'overall mood."' Their findings stand in opposition to current sentiment analysis literature, which advocates for the treatment of a sequence of messages as a single unit rather than investing in a closer analysis of how the messages relate to one another. By coding for the sentiments of each post as well as their general subject matter, our study attempted to fully capture the dynamics of both individual threads in WomenScientists1 and the forum as a whole.

There are, of course, shortcomings to these methodological approaches. Notably, social inequality creates barriers of exclusion and also allows domination by others within online spaces (Dahlberg 2001: 623). Additionally, another limitation of our methods is that it is difficult to study the interactions of this virtual community as the group is evolving. To help understand the setting in which these data are produced, we also randomly selected other forums within this life science community and then specifically observed interactions by participants of WomenScientists 1 to better contextualize their interactions within WomenScientists1. Kendall (1998) found that some virtual groups 'privilege offline identity information over information received online'. In our case, many profiles are incomplete and we only had access to forum posts and no permission to gather offline identity information.

\section{$\underline{\text { Results }}$}

Unsurprisingly, the topics addressed in the WomenScientists1 forum mostly concern the issues women face in securing and maintaining employment in the sciences. Threads varied in their degree of specificity, but mainly addressed the widespread underrepresentation of women in high-ranking positions both in research and academia, the struggles of balancing professional responsibilities and personal life, and the public perception of women in STEM and STS fields. Forum participants turned to this virtual community to interact with others who operate within the same professional and cultural paradigms, and made use of the forum to construct a community of shared norms and beliefs.

The most popular codes in the forum of WomenScientists1 are: Answers, External Source, Sex/Gender, Asks, Sex/Gender-Career, Sex/Gender Differences, Support, and Personal. 
Respectively, they were coded in the forum at a frequency of $159,148,99,98,67,63,47,40$. Noticeably, the code for 'Scientific Language' did not appear in this forum, and the relative dearth of posts coded as "science speak" or "science ethics" indicates that the primary purpose of the forum is not to initiate scientific collaboration because members do not discuss their research at length-rather, they use the forum to construct a cultural discourse that corresponds with both scientific and feminist spheres, to address the challenges of life as a female scientist (i.e. the issues that these individuals navigate). The utility of the forum differs from a strictly researchoriented approach. While the users may peripherally mention their job, they do so in a different context and for a different purpose (See Tables 1 and 2 for examples of posts).

Active users of WomenScientists1 contribute to the creation of group norms and beliefs which help satisfactorily articulate shared experiences and to begin to identify and work around common obstacles such as gender stereotypes (see Table 1) and family/work balance. Our findings expand upon the results of Brickell's analysis of online dating networks and confirm Sassen's predictions for the empowering function of virtual communities. Brickell (2012: 31) suggests that virtual forums hold constitutive power that enables individuals to decide how to identify themselves within a given community. Similarly, Sassen (2002: 379) posited that the emergence of virtual environments would allow women to participate in the creation of "a community of practice...that creates multiple lateral, horizontal communications, collaborations, solidarities, supports [... that] can enable women (or female 'subjects' generally) to pursue the projects not easily accommodated in their local, limiting and oppressive, situation". The conversations within WomenScientists1 were almost entirely geared toward finding ways to address the problems faced by women in the sciences around the world, and quickly led to the development of mentoring relationships among active users. The data collected by our application traced how these relationships emerged and suggests that the conditions of virtual forums make them particularly suited for community building among underrepresented groups.

The majority of posts to WomenScientists1 displayed either positive or ambivalent sentiments (both direct and general reference), and this trend suggests that the same is true of the entire forum. $72 \%$ of all interactions registered as either positive or neutral, and of the remainder of comments, $11.5 \%$ were unrelated to the thread, and only $16.5 \%$ were registered as negative. These findings show that WomenScientists1 is a conducive environment for the formation of supportive relationships. In instances when members did disclose personal information their 
comments almost always involved gender (see Tables 1 and 2), which sheds some light on the context and utility of the forum for users to discuss personal issues that may be unwelcome in strictly professional environments. Ross (2005) found that the Internet has become a crucial medium for the exploration of sexual identity and the enactment of sexual scripts, a trend that contributors to the forum participate in when discussing their personal lives and identities. The interactions within WomenScientists1 reveal evidence of constructive and supportive conversations, which ultimately help users feel more comfortable disclosing personal information, a finding supported by Kleinman (2003). Though the vast majority of the interactions were found to be positive or neutral, $16.5 \%$ were negative. That being said, the 'negatively' coded interactions were fairly civil and not indicative of the type of flame wars documented on the Internet. For example, one negatively coded comment read: 'suck on this [...] I have just seen an ad that has 3 women in a restaurant throw a fork on the floor so they can ogle the waiter's butt when he bends down to get it. Sexist? Definitely. Will people complain? No. If it was a waitress bending down, there would have been an outcry.' Though coded as negative per our rubric for the 'suck on this' and the quasi-troll nature of the comment, the ensuing discussion by other users was engaging and constructive with responses beginning with casual or offhand comments such as 'Having sucked on Bob's example ...', rather than a spiraling into a hostile flame war.

The first recorded contribution to the forum, posted in February 2009, mentions other sites that share the mission of WomenScientists1, contextualizing the forum within ongoing conversations about women in science. The second post cites a double standard scandal that arose that year in the UK when a female game show contestant, then-27-year-old Oxford postgraduate student Gail Trimble, was the target of negative media attention because she presented herself as an intelligent woman. The moderator of the site inquired to see what others thought about the issue and most of the thread is composed of female contributors discussing the negative portrayals of the successful UK game show contestant. This particular form of conversation acts as a type of support in that the members are able to validate their shared experiences and beliefs.

Notably, our data revealed that very few external sources were posted to WomenScientists1; Only one external source was found in initial posts, which the moderator presented through a barely noticeable hyperlink in relation to the UK game show scandal. The 
moderator wrote: "I'm assuming that the excitement over Gail Trimble, University Challenge supremo, has been mentioned to you... So what do you think about how the media and bloggers treat her?" and linked to a female science journalist's blog post on the event. "Until society accepts that we women come as we are, warts and all, girls will grow up afraid of being seen to be clever, or talented, or accomplished, for fear of being judged for their looks. Good for Gail Trimble for not allowing it to stop her," the post read.

The general lack of external sources supports our finding that information sharing is not the primary purpose of the forum, and that it is instead geared toward the exchange of opinions among individuals facing common obstacles in their personal and professional lives. When users did link to external sites, posts were aimed at self-promotion or career advancement (including links to grants and job postings). The majority of external sources posted to WomenScientists 1 provided resources. For example, a contributor posted: "I'm busy blogging but I found this very useful site: [http://rst.org/] and thought it might we worthwhile to mention here!!" The external link offered additional information to a thread about stereotype threats. In this specific example, the stereotype had to do with women in the sciences, and the link encouraged several users to provided their own links to external resources. While external sources direct users outside the forum, they do not necessarily signal diffusion of the community and are important indicators of information sharing between contributors to WomenScientists1. In their study of link-posting in online forums, Polletta et al. (2009) find that "URL-link posting not only generated more interaction than did opinions posted without links... the availability of online information may also have fostered discussions, in some instances, that were more opinionated than informed" (Polletta 2009: 1). In this forum, external sources had a similar effect-they tended to be informational and facilitated participation by reducing social capital barriers that would otherwise prevent users from interacting.

Furthermore, the fact that every question in the forum received at least one answer reveals that contributors to WomenScientists 1 actively look to respond to posts to the group, and suggests that the site has attracted a "critical mass" of contributors to make for meaningful exchanges. An EU report on establishing mentoring networks for European women found that "the presence of a critical mass of potential mentors who are willing to share their knowledge and experience is a decisive factor for the development of mentoring programmes... a critical mass of female role models is an important element for successful mentoring progammes" 
(Nöbauer and Genetti 2008: 71). Posts that did not pose questions received less traffic than those that asked something directly of forum participants, which suggests that users want to actively engage opinions within a larger conversation that extends beyond their community. Since this forum has more than one answer for every question asked and given that some topics lack any answers, users do sense that they have a distinct audience for their questions.

In one recent post, the initial question solicits opinions and seeks to elicit a discussion about how to balance a career with home life. The answers illustrate just how 'at home' forum contributors feel in this space. In this case, a more experienced poster shares her experiences and how she overcame various barriers. A second reply affirms that balancing motherhood and scientific success remains possible, and offers support for the younger scientist who posted the question. In this case, the initial poster can learn from the experiences of others while offering the replier a chance to contribute to the diversity of the field through mentorship. User interactions on WomenScientists1 lead to the evolution of different types of mentoring relationships. The use of electronic communication to foster a relationship might be considered a 'weak-tie' (Granovetter, 1973) given the lack of one-to-one direct relationships amongst the users. In other words, it might be more difficult for individuals to establish strong connections with individuals who have mentor qualities (especially experience and willingness to help). However, Packard's (2003) study of technology-supported mentoring for women in the sciences found that traditional dyadic mentoring relationships are limiting when applied to an online environment, and that networks (like WomenScientists1) amplify available resources by providing women with access to a multitude of advisers. Instead of relying on one adviser to address all of their concerns, WomenScientists 1 empowers young scientists by allowing them to consider a broad range of experiences and counsel.

Furthermore, our findings support the view that online interactions more readily lead to the sharing of personal experiences than do face-to-face $(\mathrm{FtF})$ exchanges. In a study of MentorNet, online mentoring was found to have logistical, qualitative, and managerial advantages over FtF mentoring (Mueller 2004: 57) because the online nature of the program allowed students and mentors to communicate at their convenience, and the non-verbal medium of written communications allowed them more time to develop well thought-out responses at very little cost. This is not to say that computer-mediated mentorships are always superior to 
face-to-face interactions, but simply to illustrate that they offer crucial advantages to members of underrepresented groups who are geographically separated.

\section{Conclusion}

The underrepresentation of women in the sciences is an international issue that continues to impact public policy and academic research on a large scale. While the obstacles women face when trying to establish a scientific career may not be immediately visible in their everyday lives, the "small" or "medium" biases and disadvantages that women must overcome can significantly impact their professional development (Nolan et al. 2008: 247). Likewise, though the discrepancy in the relative success of male and female scientists in the US and Europe may not immediately appear to have broad societal implications, it is indicative of systemic inequality in academia and the work force. Our findings build on the dicussion of Brickell (2012) and Arora (2012) by illuminating how virtual communities impact identity formation, contribute to the evolution of new discourses, and give voice to underrepresented groups. Brickell found that individuals turn to virtual communities as spaces in where they can 'try on' identities and eventually build a sense of self that is inflected by an existing group identity. By interacting with individuals who share common experiences and beliefs, users of WomenScientists1 formed both a group identity (as female scientists) and a common discourse (that of the challenges and inequalities faced by women in STEM/STS fields). WomenScientists1 is also demonstrative of the findings reported in this Journal by Arora (2012), which argues that virtual communities are modeled after real-world structures. Women facing 'real-world' problems - that is, institutional inequality, discriminatory selection practices, and a dearth of mentoring resources - turned to this virtual forum for support from others experiencing similar setbacks.

To prevent the persistent drop-out from the STEM/STS pipeline, it is imperative to offer these individuals opportunities to seek support from those who best understand the challenges they face in developing scientific careers. WomenScientists1 offers one such opportunity, especially because the forum connects women to specifically female mentors, and research has found that women are more likely to receive help from mentors of the same gender, who are best equipped to address both lifestyle and professional concerns (Nolan et al. 2008: 246). Single et al. (2000) and Preston (2004) highlight the problem of finding women to mentor aspiring young 
women in the sciences. As a result, students (especially women and underrepresented minorities) without this mentoring situation tend to more often leave their scientific disciplines (Herzig 2004, Nolan et al 2008, Moss-Racusin et al. 2012). While our research found that virtual communities are important mentoring resources for underrepresented groups, it is important to note that these forums are only one element of the larger process of advancing women in the sciences, and that their maximum utility comes hand-in-hand with dramatic policy measures. It should be also be noted that a limitation of our research is that we were not able to conduct interviews with members of WomenScientists1. Members from different backgrounds, with varying levels of investment in the community will likely have different views on the network. Additionally, ethnographic research could potentially highlight issues of racial and ethnic minorities in the sciences, as well as those from socioeconomically disadvantaged backgrounds, and illustrate how the members of this particular community view this intersectionality.

The primary benefit of online mentoring networks like WomenScientists1 is that they allow underrepresented groups to surmount the geographical distance between users and access a multitude of advisers. Individuals with specialized knowledge become easily accessible to others who need to find an answer to a problem they are experiencing, the virtual networks expose young scientists to experiences and possibilities well beyond the range of their local environment. As Sassen (2002: 379) observed, "of central importance for gauging the sociopolitical implications for women of their presence in, and use of, cyberspace is the potential transformation of a whole range of 'local' conditions or institutional domains where women remain the key actors, into micro-environments with global span". Online networks are crucial tools for minority groups to attain equal representation in the public sphere because they contextualize local, individually experienced issues into their broader, international context. Ultimately, WomenScientists1 and similar spaces are critical virtual communities for challenging the persistent underrepresentation of women in STEM and STS fields. 


\section{References:}

Arora, P. (2012). "Typology of Web 2.0 spheres: Understanding the cultural dimensions of social media spaces." Current Sociology 60(5): 599-618.

Abidin, C. and E. C. Thompson (2012). "Buymylife.com: Cyber-femininities and commercial intimacy in blogshops." Women's Studies International Forum 35(6): 467-477.

Bharat Mehra, C. M., and Ann Peterson Bishop (2004). "The internet for empowerment of minority and marginalized users." New Media \& Society 6(6): 781-802.

Brickell, C. (2012). "Sexuality, power and the sociology of the internet." Current Sociology 60(1): $28-44$.

Cater-Steel, Aileen and Jacquie McDonald. (2010). "Supporting Women in Engineering, Science and Technology: The Go WEST Project" In A. Cater-Steel and E. Cater (eds.), Women in Engineering, Science and Technology: Education and Career Challanges. (pp. 118137).

Chesler, N. C. \& Chesler, M. A. (2002) Gender-Informed Mentoring Strategies for Women Engineering Scholars: On Establishing a Caring Community. Journal of Engineering Education, 91, 49-55.

Cole, Jennifer. J. N., Yukari Seko, Katherine Mancuso and Alejandra Ospina (2011). "GimpGirl grows up: Women with disabilities rethinking, redefining, and reclaiming community." New Media Society 13(7): 1161-1179.

Dahlberg, L. (2001). "The Internet and Democratic Discourse: Exploring the Prospects of Online Deliberative Forums Extending the Public Sphere, Information, Communication \& Society." Information, Communication \& Society 4(4): 615-633.

Establishing Mentoring in Europe: Strategies for the promotion of women academics and researchers (2008). H. N. a. E. Genetti, eument-net: 66.

European Commission (2012). "She Figures 2012." http://ec.europa.eu/research/science society/document_library/pdf_06/she_figures_2012_en.pdf

Fay, Michaela. (2007). "Mobile Subjects, Mobile Methods: Doing Virtual Ethnography in a Feminist Online Network”. Forum: Qualitative Social Research.

Fernback, J. (2007). "Beyond the diluted community concept: a symbolic interactionist perspective on online social relations." New Media \& Society 9(1): 49-69.

Granovetter, M. S. (1973) The Strength of Weak Ties. American Journal of Sociology, 78, 13601380. 
James Hendler, N. S., Wendy Hall, Tim Berners-Lee, and Daniel Weitzner (2008). "Web Science: An Interdisciplinary Approach to Understanding the Web." Communications of the ACM 51(7): 60-69.

Herzig, A. H. (2004) Becoming Mathematicians: Women and Students of Color Choosing and Leaving Doctoral Mathematics. Review of Educational Research, 74, 171-214.

Hookway, Nicholas. (2008). 'Entering the blogosphere': some strategies for using blogs in social research. In Qualitative Research. 8(1): 91-113.

Hutchison, A. J., Johnston, L. H. \& Breckon, J. D. (2009) Using QSR-NVivo to facilitate the development of a grounded theory project: an account of a worked example. International Journal of Social Research Methodology, 13, 283-302.

Huyer, Sophia and Tatjana Sikoska. (2003). "Overcoming the Gender Digital Divide: Understanding ICTs and their Potential for the Empowerment of Women" From: (http://www.un-instraw.org/pdf/oth-Synthesis_Paper.pdf)

Kendall, L. (1998). "Meaning and Identity in "Cyberspace": The Performance of Gender, Class, and Race Online." Symbolic Interaction 21(2): 129-153.

Kennedy, T. L. M. (2000). "An Exploratory Study of Feminist Experiences in Cyberspace." Cyber Psychology 3(5): 707-720.

Kim, Soo-Min and Eduard Hovy. (2006). Extracting Opinions, Opinion Holders, and Topics Expressed in Online News Media Text. In Proceedings of the Workshop on Sentiment and Subjectivity in Text (pp.1-8). Sydney: Association for Computational Linguistics.

Kennedy, Tracey L.M.. (2000). "An Exploratory Study of Feminist Experiences in Cyberspace". CyberPsychology. 3(5): 707-720.

Kleinman, Sharon S. (2003). "Women in Science and Engineering Building Community Online”. Journal of Women and Minorities in Science and Engineering. 9(1): 73-88.

Kraut, R., Olson, J., Banaji, M., Bruckman , A. , Cohen J,. and Couper M. (2004). Psychological Research Online: Report of Board of Scientific Affairs' Advisory Group on the Conduct of Research on the Internet. American Psychologist, 59, 105-117.

Moss-Racusin, C. A., J. F. Dovidio, et al. (2012). "Science faculty's subtle gender biases favor male students." Proceedings of the National Academy of Sciences 109(41): 16474-16479.

Mueller, S. (2004) Electronic mentoring as an example for the use of information and communications technology in engineering education. European Journal of Engineering Education, 29, 53-63.

National Science Foundation, Division of Science Resources Statistics. 2011. Women, Minorities, and Persons with Disabilities in Science and Engineering: 2011. Special 
Report NSF 11-309. Arlington, VA. Retrieved July 26, 2011. Available at http://www.nsf.gov/statistics/wmpd/.

Nolan, Susan A., Cecilia H. Marzabadi, Valerie J. Kuck (2008). "Training and Mentoring of Chemists: A Study of Gender Disparity." Sex Roles 58: 235-250.

Packard, B. W.-L. (2003). "Web-based Mentoring: challenging traditional models to increase women's access." Mentoring \& Tutoring: Partnership in Learning 11(1): 53-66.

Papacharissi, Zizi. (2008). The Virtual Sphere 2.0: The Internet, the Public Sphere and beyond. Handbook of Internet Politics, Andrew Chadwick \& Philip Howard (eds.), Taylor\& Francis.

Polletta, Francesca. P. C. B. C., Christopher Anderson (2008). "Is Information Sharing Good for Deliberation? Link-Posting in an Online Forum." Journal of Public Deliberation 5(1): 120.

Preston, Anne E., (2004). "Mentoring." In Leaving Science: Occupational Exit from Scientific Careers. New York: Russell Sage Foundation. 92-111.

Robinson, L. (2007). "The cyberself: the self-ing project goes online, symbolic interaction in the digital age." New Media \& Society 9(1): 93-110.

Ross, M. W. (2005). "Typing, doing, and being: Sexuality and the internet." The Journal of Sex Research 42(4): 342-352.

Sassen, S. (2002). "Towards a Sociology of Information Technology." Current Sociology 50(3): 365-388.

Schulz, M. S. (2011). "Values and the conditions of global communication." Current Sociology 59(2): 238-251.

Single, Peg B., Carol B. Muller, Christine M Cunningham, and Richard M Single. (2000). "Electronic Communities: A Forum for Supporting Women Professionals and Students in Technical and Scientific Fields". Journal of Women and Minorities in Science and Engineering. 6(2):115-129.

Single, Peg B., Carol B. Muller, Christine M Cunningham, Richard M Single, and William S. Carlsen. (2005). "MentorNet: E-Mentoring for Women Students in Engineering and Science". Journal of Women and Minorities in Science and Engineering. 11(4): 295-309.

Stein, A. (2008). "Women Lawyers Blog for Workplace Equality: Blogging as a Feminist Legal Method." 1-50.

Thompson, E. C. (2006). "Internet-Mediated Networking and Academic Dependency in Indonesia, Malaysia, Singapore and the United States." Current Sociology 54(1): 41-61. 
Williams, J. P. and H. Copes (2005). "“How Edge Are You?" Constructing Authentic Identities and Subcultural Boundaries in a Straightedge Internet Forum." Symbolic Interaction 28(1): 67-89.

Williams, S. and Stephen Ceci. (2010). "Understanding current causes of women's underrepresentation in science." PNAS 108(8): 3157-3162.

Zorn, Isabel (2004). "Vifu: Virtual Community Building for Networking among Women" Gender, Technology. 8(1): 75-95. 